\title{
POSTOPERATIVE CHYLOTHORAX
}

Robert J. Cerfolio, MD

Mark S. Allen, MD

Claude Deschamps, MD

Victor F. Trastek, MD

Peter C. Pairolero, MD
Between July 1987 and May 1995, 11,315 patients underwent general thoracic surgical procedures at our institution. In 47 of these patients $(0.42 \%)$, postoperative chylothorax developed. There were 32 men and 15 women with a median age of 65 years (range 21 to 88 years). Initial operation was for esophageal disease in 27 patients, pulmonary disease in 13, mediastinal mass in six, and thoracic aortic aneurysm in one. All patients were initially treated with hyperalimentation, cessation of oral intake, medium chain triglyceride diet, or a combination. Nonoperative therapy was successful in 13 cases $(27.7 \%$ ), and oral intake was resumed a median of 7 days later (range 2 to 15 days). Reoperation was required in the remaining 34 cases. The reoperation rate varied according to the type of initial operation. Twenty-four of the 27 patients $(88.9 \%)$ who had undergone an esophageal operation required reoperation, versus only five of 13 patients $(38.5 \%)$ who had undergone pulmonary resection $(p<0.001)$. Lymphangiography was performed in $\mathbf{1 6}$ patients and identified the site of the leak in 13. The thoracic duct was ligated in 32 of the 34 patients who required reoperation $(94 \%)$. The remaining two patients were treated with mechanical pleurodesis and fibrin glue. Reoperation was successful in 31 of the 34 patients $(91.2 \%)$. The single death among the 47 patients $(2.1 \%)$ occurred in the reoperated group. Complications occurred in 18 patients (38.3\%). Factors that predicted the need for reoperation were initial esophageal operation and average daily postoperative drainage greater than $1000 \mathrm{ml} /$ day for 7 days. We conclude that postoperative chylothorax is an infrequent complication. Some cases can be managed without operation; however, we recommend early reoperation when drainage is greater than $1000 \mathrm{ml} / \mathrm{day}$ or if the chylous fistula occurs after an esophageal operation. The fistula can usually be controlled by ligation of the thoracic duct. (J Thorac Cardiovasc Surg 1996;112:1361-6)
$T_{p}$ he thoracic duct, the main tributary of the lymphatic system, is a 2.0 to $3.0 \mathrm{~mm}$ thin-walled structure that begins at the cisterna chyli at the second lumbar vertebra and enters the chest through the aortic hiatus. It lies posterior to the aorta and courses to the right of the spine. It usually crosses to the left at

From the Section of General Thoracic Surgery, Mayo Clinic and Mayo Foundation, Rochester, Minn.

Read at the Seventy-sixth Annual Meeting of The American Association for Thoracic Surgery, San Diego, Calif., April 28-May 1, 1996.

Received for publication May 6, 1996; revisions requested June 18, 1996; revisions received July 22, 1996; accepted for publication July 26, 1996.

Address for reprints: Mark S. Allen, MD, Department of Surgery, Mayo Clinic, 200 First Street S.W., Rochester, MN 55905.

Copyright (ㅇ) 1996 by Mosby-Year Book, Inc.

$0022-5223 / 96 \$ 5.00+0 \quad \mathbf{1 2 / 6 / 7 6 9 1 8}$ the fifth thoracic vertebra and then ascends into the neck posterior to the aortic arch and empties into the left jugulosubclavian junction. Its proximity to the esophagus, presence of collateral channels, and highly variable course leads to its injury during esophageal and pulmonary resection. Injuries to the main thoracic duct or large tributaries rarely close spontaneously. We reviewed our experience with iatrogenic chylothorax after general thoracic surgical procedures to identify factors that predict the need for reoperation and to evaluate the success of management.

\section{Patients and methods}

Between July 1987 and May 1995, 11,315 general thoracic surgical procedures were performed at our institution and a postoperative chylothorax developed in 47 patients $(0.42 \%)$. The diagnosis was established by a triglyceride content of 110 $\mathrm{mg} / \mathrm{dl}$ or greater and the presence of chylomicrons in the pleural fluid in all patients. 
Table I. Initial operation

\begin{tabular}{lc}
\hline & $\begin{array}{c}\text { No. of } \\
\text { patients }\end{array}$ \\
\hline Esophageal operations & \\
Ivor Lewis esophagogastrectomy & 10 \\
Extended Ivor Lewis esophagogastrectomy & 6 \\
Transhiatal esophagogastrectomy & 6 \\
Transhiatal esophagogastrectomy/total laryngectomy & 2 \\
Esophagectomy with colonic interposition & 1 \\
Esophageal myotomy & 1 \\
Uncut Collis-Nissen gastroplasty & 1 \\
& 27 \\
Pulmonary resections & 8 \\
Lobectomy & 2 \\
Wedge excision & 1 \\
Segmentectomy & 1 \\
Pneumonectomy & 1 \\
Sleeve pneumonectomy & 13 \\
Others & \\
Resection of mediastinal mass & 6 \\
Repair descending thoracic aortic aneurysm & 1 \\
& 7
\end{tabular}

The medical records of these 47 patients were reviewed for age, sex, preoperative history, operative and pathologic findings, amount of daily chest tube drainage, and method of management of the chylothorax. Lymphangiography was performed by direct cannulation of a lymphatic channel in the dorsum of the foot after injection of Ethiodol (Altana, Inc., Melville, N.Y.) into the four webbed spaces on each foot. After cannulation of a lymphatic channel, 1\% Lymphazurin (isosulfan blue; Hirsch Industries, Inc., Richmond, Va.) contrast was injected and serial roentgenograms were performed. Follow-up data were obtained from the patients' most recent clinic visits, home health care providers, and, when needed, telephone interviews.

Patients with cancer were postsurgically staged by the TNM classification system of the American Joint Committee on Cancer Staging and End Results Reporting. ${ }^{1}$ Operative mortality was defined as any death within 30 days of operation or during the same hospitalization. Survival was estimated by the Kaplan-Meier method with the date of thoracotomy as the starting time. ${ }^{2}$ The influences of variables on survival and morbidity were analyzed with the proportional hazards model of $\mathrm{Cox}^{3}$ for continuous variables and the log-rank test for discrete variables. ${ }^{4}$ Values of $p$ lower than 0.05 were considered statistically significant. Data are expressed as medians with ranges.

\section{Clinical findings}

There were 32 male and 15 female patients, with a median age of 65 years (range 21 to 88 years). Significant medical conditions were present before initial thoracotomy in 25 patients $(53.0 \%)$ and included myocardial infarction in seven, hypertension in five, chronic obstructive pulmonary disease in four, lymphoma in three, diabetes mellitus in two, and atrial fibrillation, colon cancer, breast cancer, and lung cancer in one each. Three patients had previous contralateral thoracotomy and two had previous ipsilateral thoracotomy. The indications for the five previous thoracotomies were pulmonary metastases in two patients and gastroesophageal reflux, empyema thoracis, and lung cancer in one patient each. Preoperative radiation therapy was administered to six patients, and five received preoperative chemotherapy.

The initial operations were performed for esophageal cancer in 23 patients, lung cancer in 10 , mediastinal mass in six, pulmonary metastases in two, reflux esophagitis in two, benign esophageal stricture in two, and achalasia and thoracic aortic aneurysm in one each.

\section{Results}

The initial procedures performed are shown in Table I. Esophageal operations were performed in 27 patients, pulmonary in operations were performed 13 , and other operations were performed in seven.

All patients had thoracostomy tubes after their initial operation. Two patients who had a pneumonectomy had the chest tube removed immediately after skin closure in the operating room. Chylothorax was diagnosed a median of 5 days after operation (range 3 to 82 days). The chylothorax was on the right side in 34 patients, on the left side in nine, and bilateral in four. The mean triglyceride content of the drainage was $370 \mathrm{mg} / \mathrm{dl}$ (range 118 to 2416 $\mathrm{mg} / \mathrm{dl}$ ), and the mean cholesterol content of the fluid was $48 \mathrm{mg} / \mathrm{dl}$ (range 19 to $97 \mathrm{mg} / \mathrm{dl}$ ). All patients had chylomicrons in their chest drainage. The median daily chest drainage for the first 7 postoperative days was $1117 \mathrm{ml}$ (range 286 to 2225 $\mathrm{ml}$ ). All patients were initially treated by dietary manipulation. This included complete cessation of oral intake and central hyperalimentation in 36 patients and a medium-chain triglyceride diet in 11 patients. This treatment was successful for seven patients who received total parenteral nutrition and in six patients who received medium-chain triglyceride diets. These patients resumed a normal diet a median of 7 days after the diagnosis of chylothorax was made (range 2 to 15 days). The remaining 34 patients $(72.3 \%)$ required reoperation to control the fistula. The median daily chest drainage for the first 7 postoperative days was $1168 \mathrm{ml}$ in the group that required reoperation and $511 \mathrm{ml}$ for the patients who did not require reoperation (Fig. 1). Additionally, 24 of the 27 patients in whom the chylothorax 


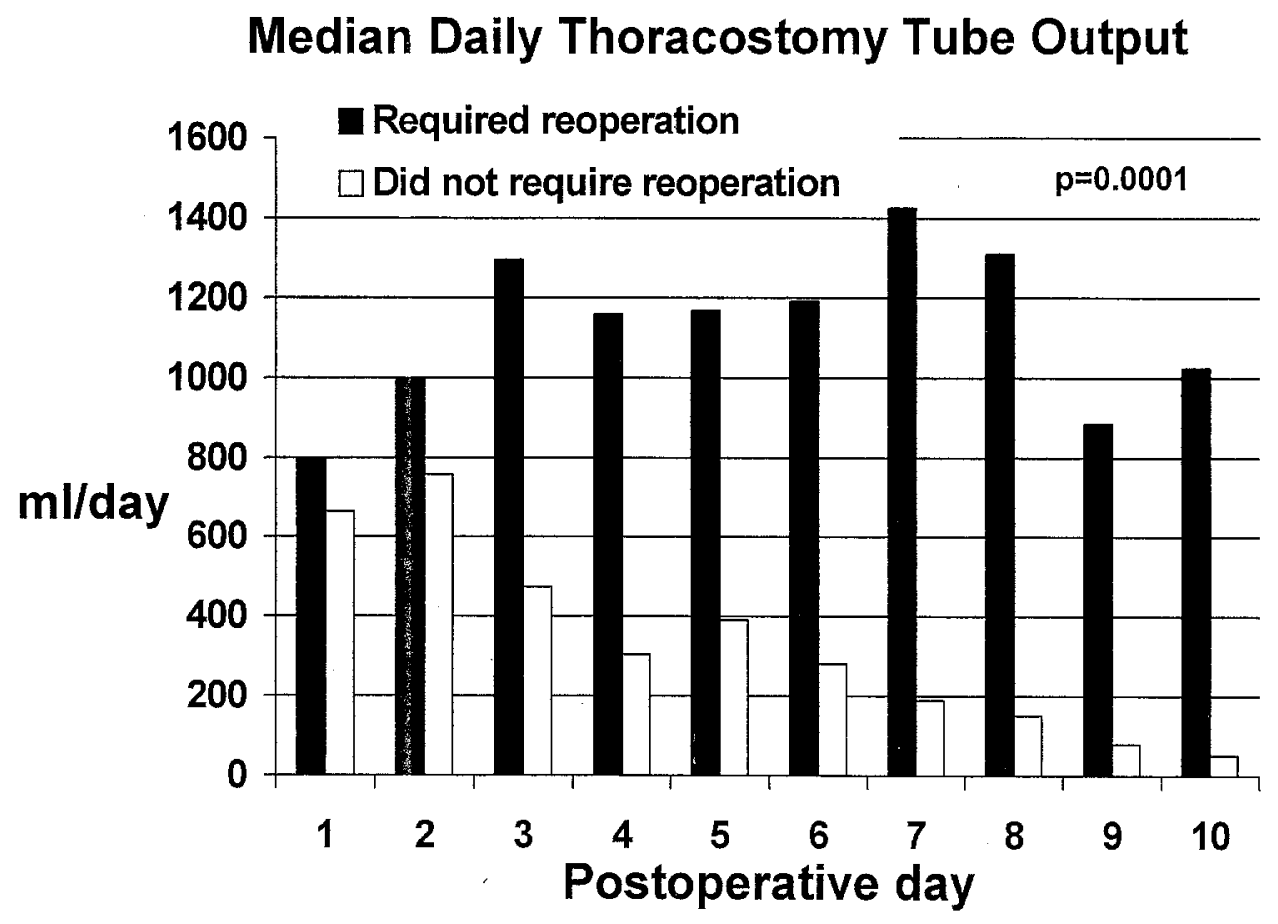

Fig. 1. Comparison of median daily thoracostomy tube output of 34 patients who required reoperation and 13 patients who did not. Median daily thoracostomy output was statistically significantly different between these two groups $(p=0.0001)$.

occurred after esophageal operations $(88.9 \%)$ required reoperation to control the fistula; only five of 13 who had chylothorax after pulmonary resections $(38.5 \%)$ required reoperation $(p<0.001)$. Lymphangiography was performed in 16 patients before reoperation and a leak was identified in $13(81.3 \%)$. In these 13 patients, reoperation confirmed this area to be the site of the fistula.

Reoperation was undertaken a median of 9 days after operation (range, 4 to 106 days). The reoperation was performed on the side of the chylothorax in 28 patients and on the contralateral side in six. The approach for the reoperation was through the initial thoracotomy in 28 patients, through a new thoracotomy in three, and by video-assisted thoracic surgery in three. Two of the patients who underwent videoassisted thoracoscopic surgery had the procedure converted to thoracotomy because of inability to visualize the duct. The injury to the thoracic duct was identified at reoperation in 23 of the 34 patients $(67.6 \%)$ and was at the level of the diaphragm in 11 , the azygos vein in nine, and the apex of the chest in three.

The thoracic duct was ligated in 32 of the 34 patients. The site of ligation was the diaphragm in
13 patients, immediately below the fistula in 12 , and both sites in seven. In addition, mechanical pleurodesis was performed in 16 , talc pleurodesis was performed in three, and a partial parietal pleurectomy was performed in three. The two patients that did not have the thoracic duct ligated underwent mechanical pleurodesis and had fibrin glue applied to the posterior mediastinum. The fistula was successfully controlled in 31 patients $(91.2 \%)$. Three patients required a second reoperation because of continued leakage of chyle; the second reoperation was successful in all three cases. Table II illustrates the management of these cases.

There was one death among the 47 patients $(2.1 \%)$. This death was that of a 70-year-old man with severe chronic obstructive pulmonary disease who underwent a transhiatal esophagectomy for $\mathrm{T}_{3} \mathrm{~N}_{0} \mathrm{M}_{0}$ squamous cell cancer of the esophagus. He had a left thoracotomy for chylothorax on postoperative day 8 . Pneumonia developed, and he died of sepsis and adult respiratory distress syndrome. Complications occurred in 18 patients (38.3\%): atrial fibrillation in 12 , prolonged ventilation in three, and prolonged air leak, cervical anastomotic leak, and respiratory failure necessitating tracheostomy in one each. 
Table II. Management of three patients who had multiple reoperations to control chylothorax

\begin{tabular}{ccc}
\hline \multicolumn{1}{c}{ Initial reoperation } & Second reoperation \\
\hline 1 & $\begin{array}{c}\text { Ligated duct at diaphragm } \\
\text { and fibrin glue applied to } \\
\text { mediastinum }\end{array}$ & $\begin{array}{c}\text { Religated duct at dia- } \\
\text { phragm, pleurectomy, } \\
\text { and fibrin glue applied to } \\
\text { mediastinum }\end{array}$ \\
$\begin{array}{l}\text { Ligated duct at level of azy- } \\
\text { gos vein and talc pleu- } \\
\text { rodesis } \\
\text { Ligated duct at diaphragm }\end{array}$ & $\begin{array}{l}\text { Ligated duct in abdomen } \\
\text { via laparotomy }\end{array}$ \\
& $\begin{array}{l}\text { Ligated accessory thoracic } \\
\text { duct }\end{array}$ \\
\hline
\end{tabular}

\section{Discussion}

Chylothorax is uncommon after thoracic surgical procedures. In our review, we found only 47 patients with chylothorax out of 11,315 who underwent a general thoracic surgical procedure at our institution $(0.42 \%)$. Although chylothorax has been reported after almost every type of thoracic operation, certain patients may be predisposed toward postoperative chylothorax. Esophageal resections for carcinoma with mediastinal lymphadenectomy may lead to a higher incidence of postoperative chylothorax. Among our patients, the overall incidence of chylothorax was $0.42 \%$; after esophagectomy, however, it was $2.9 \%$. In contrast, our incidence of postpneumonectomy chylothorax was much lower $(0.37 \%)$ and compares favorably with that reported by Vallieres, Shamji, and Todd ${ }^{5}$ in 1993. Our incidence of chylothorax after lobectomy was $0.26 \%$. We believe that intraoperative prevention is important. If the thoracic duct is suspected to have been damaged during a thoracic procedure, ligation, pleurodesis, or both should be performed to prevent subsequent chylothorax.

Injury to the thoracic duct should be suspected when unexplained high thoracostomy tube drainage is present after operation. The fluid may not be "milky white" if the patient is not taking anything by mouth. The diagnosis of chylothorax is established by examination of pleural fluid. Both chylomicrons and a triglyceride concentration greater than 110 $\mathrm{mg} / \mathrm{dl}$ are diagnostic of chylothorax. After pneumonectomy, the diagnosis is more difficult because fluid normally fills the postpneumonectomy space. However, patients with a chylothorax after pneumonectomy often have a shift of the mediastinum to the contralateral side because of continued infusion. If chylothorax is suspected, thoracentesis should be performed to establish the diagnosis.

The electrolyte content of chyle is similar to that of serum. ${ }^{6}$ Because the thoracic duct transports $60 \%$ to $70 \%$ of ingested fat to the blood stream, the usual concentration of fat in chyle is 0.4 to $6.0 \mathrm{gm} / \mathrm{dl}$. The concentration of protein in chyle is 2.2 to $6.0 \mathrm{gm} / \mathrm{dl} .^{7}$ Chyle also contains from 400 to 6800 white blood cells $/ \mathrm{ml}$.

The thoracic duct flow increases after meals, especially when a high-fat meal is ingested. Flow rates as high as $110 \mathrm{ml} / \mathrm{hr}$ have been recorded. From 2 to $3 \mathrm{~L}$ chyle, with its associated proteins, white blood cells, and electrolytes, can be lost daily from a thoracic duct fistula. Consequently, a prolonged chyle fistula is poorly tolerated and is associated with significant mortality if not corrected.

Because starvation usually reduces the flow of chyle, all of our patients had complete cessation of oral intake after a chylous fistula was diagnosed. In selected patients, a medium-chain triglyceride diet also may be successful. The theory behind a medium-chain triglyceride diet as a treatment for chylous fistula was described in 1964 by Hashim and colleagues. ${ }^{8}$ These triglycerides contain fatty acids with a chain length of fewer than 12 carbon atoms and are absorbed into the portal vein and transported directly to the liver, where they are metabolized. Six of our 11 patients who were placed on a medium-chain triglyceride diet had resolution of their chylothorax.

If nonoperative therapy with cessation of oral intake or a medium-chain triglyceride diet is unsuccessful, reoperation should be considered. Identification of the site of the chyle leak can help to determine the surgical approach, allowing the surgeon to look in a specific area. Lymphangiography successfully located the leak in $81 \%$ of our patients. In addition, lymphangiography can demonstrate accessory ducts and the course of the main duct. None of our patients had pulmonary dysfunction as a result of lymphangiography, as was reported by Rosenow, Wilson, and Cockerill. ${ }^{9}$

Other methods that may help identify the site of leak at operation include feeding the patient a high-fat diet several hours before operation. This will increase the flow of chyle and can assist the surgeon in identifying the leak. In 1955, Merrill ${ }^{10}$ described injection of Evans blue dye subcutaneously in the thigh for prompt intraoperative identification of the thoracic duct. A year later, Stranahan and colleagues ${ }^{11}$ described intraoperative ductography to aid in identifying the fistula. Neither of these latter techniques was employed in any of our cases.

The decision of when to reoperate is important. 
We found that chest thoracostomy drainage greater than $1000 \mathrm{ml} /$ day for the first 7 days and the development of a chylothorax after an esophageal operation were significant factors that predicted the need for reoperation. Others have reported similar findings. ${ }^{12}$ Patterson and coworkers ${ }^{13}$ recommended observation for 7 days; if drainage was still greater than $1000 \mathrm{ml} /$ day, reoperation to ligate the thoracic duct was necessary. If drainage is high or chylothorax occurs after an esophageal operation, early reoperation should be strongly considered.

The reoperative approach is another important decision. In most of our patients with a previous thoracotomy, the chylothorax was on the ipsilateral side and the reoperation was through the original thoracotomy. If lymphangiography demonstrates that the thoracic duct is only accessible from the contralateral side, however, a contralateral thoracotomy should be performed.

When the reoperation is performed on the side of the chylothorax, pleurodesis or pleurectomy should also be performed at the same time as thoracic duct ligation. If the thoracic duct is ligated contralateral to the effusion, pleurodesis or pleurectomy is not necessary.

In most of our patients, the thoracic duct was ligated at the diaphragmatic level, as suggested by Murphy and Piper ${ }^{14}$ in 1977. Ligation at this level has the advantage of stopping flow from any accessory ducts that may not be recognized. If the thoracic duct cannot be found or if ligation does not control the leak, pleurodesis is indicated. Other methods to control chylothorax described in the literature include radiation therapy, phrenic nerve crush, and reimplantation of the thoracic duct into a vein or reanastomosis of a torn thoracic duct. ${ }^{5}$ Finally, a pleural-peritoneal shunt can be used to transport chyle into the abdomen or a pleuralvenous shunt can be employed to reinfuse chyle into the venous system. Readministration of aspirated chyle by mouth or rectum has been tried but has not been found to be of benefit. None of these alternative techniques were used in our patients.

Chylothorax is an infrequent postoperative complication. Although chylothorax can occasionally be treated by nonoperative therapy, when chest thoracostomy drainage is greater than $1000 \mathrm{ml} /$ day or chylothorax occurs after an esophageal operation, surgical intervention will probably be necessary. Lymphangiography can help locate the fistula and determine the course of the thoracic duct. The fistula can usually be controlled by ligation at the diaphragm or at the site of the fistula. Pleurodesis may also be necessary.

\section{REFERENCES}

1. Beahrs OH, Myers MH, editors. American Joint Committee on Cancer: manual for staging of cancer. 2nd ed. Philadelphia: Lippincott, 1983:178.

2. Kaplan EL, Meier P. Non-parametric estimation from incomplete observations. J Am Statist Assoc 1958;53:457-81.

3. Cox DR. Regression models and life-table (with discussion). J R Statist Soc Series B 1972;34:187-220.

4. Peto R, Peto J. Asymptotically efficient rank and invariant procedures. J R Statist Soc, Series A 1972;135:185-201.

5. Vallieres E, Shamji FM, Todd TR. Postpneumonectomy chylothorax. Ann Thorac Surg 1993;55:1006-8.

6. Robinson CL. The management of chylothorax. Ann Thorac Surg 1985;39:90-5.

7. Bessone LN, Ferguson TB, Burford TH. Chylothorax. Ann Thorac Surg 1971;12:527-50.

8. Hashim SA, Roholt HB, Babayan VK, Van Itallie TB. Treatment of chyluria and chylothorax with medium-chain triglyceride. N Engl J Med 1964;270:756-61.

9. Rosenow EC, Wilson WR, Cockerill FR. Pulmonary disease in the immunocompromised host (first of two parts). Mayo Clin Proc 1985;60:473-87.

10. Merrill $\mathrm{K}$ Jr. The use of Evans blue to outline the course of the thoracic duct. J Thorac Cardiovasc Surg 1955;29:555-7.

11. Stranahan A, Alley RD, Kausel HW, Reeve TS. Operative thoracic ductography. J Thorac Cardiovasc Surg 1956;31:18398.

12. Selle JG, Snyder WH, Schreiber JT. Chylothorax: indications for surgery. Ann Surg 1973;177:245-9.

13. Patterson GA, Todd TR, Delarue NC, Ilves R, Pearson FG, Cooper JD. Supradiaphragmatic ligation of the thoracic duct in intractable chylous fistula. Ann Thorac Surg 1981;32:44-9.

14. Murphy TO, Piper CA. Surgical management of chylothorax. Am Surg 1977;43:715-8.

\section{Discussion}

Dr. George T. Christakis (Toronto, Ontario, Canada). Congratulations, on a well-presented and well-thought out article. This is an important study because it expands the surgeon's knowledge and understanding of an uncommon complication after thoracic surgery.

Current trends in the epidemiology of disease have underscored the necessity of using evidence-based medicine when producing conclusions or recommendations from studies. This would normally impose the need for a prospective randomized trial to reasonably answer any questions. In the case of rare postoperative outcomes, such as the incidence of chylothorax following thoracic surgery, a randomized control trial would require the enrollment of many thousands of patients and would be practically impossible. This represents the logic for my praise, and for soliciting further presentations that address rare perioperative complications.

On the basis of this large experience from the Mayo Clinic, I would like to ask the following questions. First, patients undergoing operations for esophageal cancer have a limited long-term survival, and the major indication for surgery is improvement of the quality of life. 
Because you have clearly demonstrated that $89 \%$ of patients with chylothorax after esophageal surgery eventually require repair, why extend costly hospital stay by treating these patients with hyperalimentation? Why not recommend immediate operation on discovering chylothorax? This would expedite recovery and minimize hospital stay, so that patients would be able to live whatever life they had left outside of hospital.

Second, medium-chain triglyceride diets appeared to have a high success rate, certainly higher than parenteral nutrition in this study. What were the indications for prescribing a medium-chain triglyceride diet, and why do you not recommend that all patients with chylothorax be given such a diet?

Finally, lymphangiograms also appeared to be very successful in identifying the site of the leak in 13 of 16 patients in your study. Why were lymphangiograms not performed routinely for all patients, especially because this test may find a leak in the contralateral side of the chest?

Dr. Cerfolio. We recommend waiting 1 week before reoperation in patients with chylothorax after esophageal resection for several reasons. First, the diagnosis of chylothorax is usually not made until approximately 4 or 5 days after operation. It is only suspected in the first few postoperative days because of unexplained high chest tube drainage. It then takes a few more days for the lipid analysis to confirm the diagnosis. Because we have shown that there is about $10 \%$ success with medical treatment, we believe a medical trial of a few days is warranted, especially in patients with drainage of $1000 \mathrm{ml}$ or less. Furthermore, a few days gives the patients time to both prepare physically and mentally for reoperation. Past literature has shown that a 3- to six-week delay is associated with significant morbidity, but we do not believe that this is true of a 2- to 3-day delay.

Your second question concerned medium-chain triglyceride diet. We had 11 patients who received mediumchain triglyceride diets and 34 who got total parenteral nutrition. As you know, this is a retrospective study. Seven of 11 patients who received medium-chain triglyceride diets did not have esophageal resections, they had pulmonary resections, and they had drainage that was on average lower than that of the patients who received total parenteral nutrition. The success rate may have been higher for patients receiving medium-chain triglyceride diets because of patient selection. We do not believe that the medium-chain triglyceride diet is superior to total parenteral nutrition.

Your third question concerned lymphangiography. There are four thoracic surgeons at the Mayo Clinic, and they all have different practices. One surgeon routinely uses lymphangiography for patients with postoperative chylothorax, and he has found it to be valuable; however, he has not quite persuaded the other three. I believe that this article does offer evidence that lymphangiography is a valuable test. It helps to identify accessory ducts, which are not always controlled when the main thoracic duct is ligated. Second, and very important, it identifies the main thoracic duct and guides the surgeon's reoperative approach for ligation.

Dr. Joseph I. Miller (Atlanta, Ga.). While I was at the Mayo Clinic, O. T. Clagett stated that if you had a postoperative thoracic duct fistula you could radiate the thoracic duct to 2000 rads. In my 23 years at Emory, we have had four such cases, two after an Ivor Lewis esophagogastrectomy and one right pneumonectomy and one left upper lobectomy. We added 2000 rads after operation, and they all stopped. I know of six other cases in the southern region in which the fistula, stopped, as Clagett's advice suggests. Only in the case of malignant disease, but you can radiate the thoracic duct to about 2000 to 2500 rads and they all stop without reoperation.

Dr. Cerfolio. We do mention some of the alternative treatments for chylothorax in the article. We believe that there is a role for some of these in selected patients. In patients with benign disease, radiation would probably be a poor choice. We appreciate your bringing up the option of radiation for patients with malignant disease.

Dr. Anthony L. Moulton (Providence, R.I.). Just a couple of questions. In terms of the esophageal disease, did you see a difference in the incidence of chylothorax between patients who underwent a transhiatal resection and those who underwent an Ivor Lewis resection? What was the effect of the extent of the nodal dissection as well?

Recently we have been involved with a number of patients who have received preoperative paclitaxel (Taxol) and irradiation. In those patients, we have found an incredible inflammatory response with massive lymphadenopathy. We have felt obligated to resect all of that to make sure that there was no residual tumor. It is our impression that we have seen an increased incidence of chylothorax after this extensive dissection. Do you have any comments about the incidence of chylothorax in patients who received neoadjuvant therapy before operation?

Dr. Cerfolio. Those are important questions that we did statistically analyze. Because of the small numbers in this series, we cannot say that one type of esophageal resection had a higher incidence of postoperative chylothorax than another. Also, we found no statistically significant difference between patients with positive lymph nodes versus those with negative lymph nodes. One would need to analyze all patients who underwent esophagogastrectomy, however, and compare the incidences of postoperative chylothorax among the patients with positive lymph nodes and those with negative lymph nodes to accurately answer your question.

At the Mayo Clinic, the general philosophy is to do a rather large resection of tissue around the esophagus, and oftentimes the thoracic duct is seen during operation. If the duct is seen at the time of initial esophageal resection, it is prophylactically ligated at the level of the diaphragm. 